\title{
Gluten Sensitivity in Pediatrics: A Clinical Conundrum
}

\author{
Fernanda Cristofori • Francesca Arezzo • \\ Antonia Gentile $\cdot$ Ruggiero Francavilla
}

Published online: 1 August 2014

(C) Springer Science + Business Media New York 2014

\begin{abstract}
Non-celiac gluten sensitivity (NCGS) is a condition in which symptoms are triggered by gluten ingestion, in which both allergic and autoimmune mechanisms have been ruled out. The "classical" presentation of NCGS is a combination of gastrointestinal symptoms (including abdominal pain, bloating, and bowel habit abnormalities) and extra-intestinal manifestations. So far, NCGS has been described extensively in adults, and few data are available for the pediatric population. Due to lack of a serological or histological marker, the diagnosis of NCGS remains clinical and it is based on the exclusion of celiac disease and wheat allergy and on gluten challenge. $G$ is a 2.5-year-old child referred to our Center for chronic diarrhea, diurnal abdominal pain, and mild failure to thrive. His mother reported that symptoms were related to wheat ingestion and that resolved on a gluten-free diet; therefore, she was worried about Celiac Disease. However, blood tests did support neither celiac disease because of antitransglutaminase-IgA, anti-endomysium, and anti-gliadinIgA negativity nor wheat allergy for the absence of specific IgE for wheat or gluten. $G$ was positive just to anti-gliadinIgG. We reassured the mother and concluded for a chronic unspecific diarrhea to be treated conservatively without elimination diets. On the follow-up visit, $G$ was still complaining with same symptoms, and his growth was further slowing; we ,therefore, decided to repeat the blood tests (with similar results), to define the HLA status (DQ1/
\end{abstract}

F. Cristofori · F. Arezzo · A. Gentile · R. Francavilla Pediatric Section, Interdisciplinary Department of Medicine,

University of Bari, Bari, Italy

R. Francavilla $(\bowtie)$

Clinica Pediatrica "B. Trambusti"-Giovanni XXIII Hospital,

Via Amendola 270, Bari, Italy

e-mail: rfrancavilla@me.com
7), and to perform intestinal duodenal biopsies. These showed normal villous architecture and a modest increase of intraepithelial lymphocytes. Based on the clear association between clinical symptoms and wheat ingestion, the final diagnosis was "non IgE-mediated adverse reaction to wheat," although G had no other clue to support this diagnosis such as personal or family history of atopy and/or eczema. $\mathrm{G}$ was started on a gluten-free diet with resolution of symptoms. This diagnosis was confirmed 6 months later with a double-blind placebo-controlled challenge that resulted in the onset of diarrhea and abdominal pain $30 \mathrm{~h}$ after wheat ingestion. This was happening in 2006 and 2 years later a "new" disease entity might have better explained the symptoms of this child: NCGS.

Keywords Non-celiac gluten sensitivity - Abdominal pain $\cdot$ Pediatric gastroenterology

$\begin{array}{ll}\text { Abbreviations } \\ \text { AGA } & \text { Anti-gliadin } \\ \text { APT } & \text { Atopy patch test } \\ \text { ATIs } & \text { Alpha-amylase trypsin inhibitors } \\ \text { CD } & \text { Celiac disease } \\ \text { EMA } & \text { Anti-endomysial } \\ \text { DBPCFC } & \text { Double-blind placebo-controlled food } \\ & \text { challenge } \\ \text { FODMAPs } & \text { Fermentable oligo-, di-, and mono- } \\ & \text { saccharides and polyols } \\ \text { GFD } & \text { Gluten-free diet } \\ \text { NCGS } & \text { Non-celiac gluten sensitivity } \\ \text { IBS } & \text { Irritable bowel syndrome } \\ \text { IELs } & \text { Intraepithelial lymphocytes } \\ \text { WA } & \text { Wheat allergy } \\ \text { SPT } & \text { Skin prick test } \\ \text { TTG } & \text { Anti-transglutaminase }\end{array}$




\section{Introduction}

The possibility of a causal relationship between the ingestion of gluten and development of symptoms in the absence of celiac disease (CD) and wheat allergy (WA) has long been ignored, but recently has gained new scientific interest expanding beyond these well-recognized disorders and now including a new entity known as non-celiac gluten sensitivity (NCGS) [1, 2••].

This entity had firstly been described more than 30 years ago in eight adult females suffering from abdominal pain and chronic diarrhea which had dramatic relief on a glutenfree diet (GFD) and return of symptoms after gluten challenge [3]. In the same years, Ellis and Linaker [4] described normal biopsies and normal lymphocyte counts existing in combination with rapid disappearance of symptoms when their patients withdrew gluten from the diet.

Recently, the gluten-free market increased worldwide, partially sustained by a percentage of general population who consider themselves suffering from "problems caused by gluten ingestion" and who undergo a GFD without having a proper diagnosis. Paradoxically in US population, more people are aware of NCGS than they are of CD [5]. Nowadays, marketers estimate that 15 to $25 \%$ of North American consumers want gluten-free foods, although doctors estimate that just $1 \%$ have $\mathrm{CD}$ [6] although recently published data would suggest this to be an overestimation [7].

The first recent description of NCGS has been reported by Sapone et al. [8], while the existence of this condition has been confirmed by Biesiekierski et al. [9•] in 2011, who demonstrated for the first time that individuals with a history of gluten sensitive symptoms did indeed respond to a double-blind gluten challenge. Since then, several reports from different groups have been published. A large Italian retrospective study on 920 adult patients with irritable bowel syndrome (IBS) demonstrated that $30 \%$ were positive to a wheat double-blind placebo-controlled food challenge (DBPCFC) and therefore classified as NCGS (74\% suffering from multiple food sensitivities) [10•]. Finally, a recent study on patients with diarrhea predominant IBS suggested that gluten withdrawal improved stool frequency, while its administration negatively influenced intestinal permeability [11].

So far, NCGS has been extensively described in adults, while little evidence is available in pediatric age; however, in children, NCGS might have been diagnosed as non-IgEmediated adverse reaction to wheat. Our group described for the first time NGCS in 15 children referred for a clear association between gluten consumption and symptoms supporting the existence of this condition across all ages with clinical, serological, genetic, and histological features partially similar to those reported in adults [12••].

\section{Definition}

Gluten sensitivity is a condition in which symptoms are triggered by gluten ingestion, and where both allergic and autoimmune mechanisms have been ruled out (diagnosis by exclusion criteria). Patients should have negative celiac serology, negative histology for classical celiac villous atrophy, and negative $\operatorname{IgE}$ based tests. In order to avoid confusion with $\mathrm{CD}$, sometimes defined as gluten-sensitive enteropathy, "non celiac gluten sensitivity" appeared as an improved definition [1, 2••]. As triggering cereal proteins could include fractions other than gluten, some authors argue in favor of "non-celiac wheat sensitivity" [13], a terminology that would, however, conflict with the possibility that other gluten-containing cereals (rye, barley) may be offensive for the "gluten sensitive" patient, an unexplored area.

\section{Epidemiology}

The prevalence of NCGS is not yet established, although it is commonly thought to be at least as common as CD and likely much more prevalent in adults than in children. It is estimated that up to $6 \%(347 / 5.896$ patients seen at the Center for Celiac Research, University of Maryland) of the general population might be affected [1].

Tanpowpong et al. [7] reported that $5 \%$ of children without $C D$ avoid gluten-containing food, and that this dietetic restriction in a different cohort is associated with improvement of non-specific behavioral and gastrointestinal complaints [14]. However, it is unclear how many children reporting gluten avoidance were indeed affected by NCGS, as the vast majority of the children were neither tested for CD nor underwent an intestinal biopsy.

A recent population-based survey in 7,762 unselected US persons (aged 6 years or older participating to the National Health and Nutrition Examination Survey) responding to an interviewer-administered questionnaires showed that $0.55 \%$ were following a gluten-free diet, suggesting NCGS to be affecting at most $0.55 \%$ of the general population. [15].

Finally, Aziz et al. [16] on a population of 1,002 adults describe a self-reported prevalence for NCGS of $13 \%$ (female $79 \%$ ) with $3.7 \%$ consuming a gluten-free diet and $0.8 \%$ known to have a doctor diagnosis of $\mathrm{CD}$; individuals with NCGS had an increased prevalence of fulfilling the Rome III criteria for IBS, in comparison with those without NCGS.

\section{Pathogenesis}

The pathogenesis of this condition is still debated, although recent data suggest a major role for abnormalities in the 
innate immune system. Sapone et al. compared small intestinal biopsies of patients with CD and NCGS showing that intestinal permeability was intact in patients with NCGS, unlike patients with CD. The author reports that unlike CD, NCGS is associated with an increased expression of the innate immunity marker (Toll-like receptor 2) a reduction of marker FOXP3 and no alterations of adaptive immunity markers (interleukin -6 and -21 ) suggesting that NCGS is associated with prevalent activation of an innate immune response responsible for the loss of gluten tolerance [17].

Recently, Brotveit et al. [18] showed that NCGS patient had and increased density of intraepithelial CD3(+) T cells at baseline and a significant increase in interferon- $\gamma$ mRNA following gluten challenge suggesting that the adaptive immune system may play a role in NCGS.

Animal models of GS may help to explain the genesis of GI symptoms in the absence of intestinal damage. Gluten sensitization of DQ8 mice increases acetylcholine release by the myenteric plexus after in vitro nerve stimulation. The increased acetylcholine release is accompanied by hypercontractility and dysmotility, characterized by the increased incidence of retroperistalsis and altered gastric emptying [19].

The real trigger of NCGS is still to be defined. Gluten cannot be considered the sole agent responsible for symptoms and other proteins, such as-amylase/trypsin inhibitor (ATI) or even yeast, may be involved; in addition, some of the carbohydrates escape small-bowel digestion and absorption, and may cause abdominal discomfort and diarrhea. Moreover, the trigger/s of mucosal events leading to NCGS may not be necessarily represented by the same array of gluten peptides responsible for the $\mathrm{CD}$ development. In NCGS patients, gliadin fractions do not provoke a clear picture of inflammation upon contact with the duodenal mucosa or with peripheral basophils [20]. In vitro studies suggest that wheat ATIs could play a major role as triggers of the innate immune response in GS [21].

In 2013, Biesiekirski et al. [22] provided evidence that carbohydrates may be responsible for symptoms thought to be related to NCGS. They reported 37 patients with IBS/ self-reported NCGS who were assigned to a period of reduced low-fermentable, poorly absorbed, short-chain carbohydrates (fermentable oligo-, di-, and mono-saccharides and polyols $=$ FODMAPs) diet, and then placed on either a gluten or whey proteins challenge. In all participants, gastrointestinal complaints consistently improved during reduced FODMAP intake but significantly worsened to a similar degree when their diets included gluten or whey proteins [22]. FODMAPS list includes fructans, galactans, fructose, and polyols that are contained in several foodstuffs, including wheat, vegetables, and milk derivatives. These results raise the possibility that the positive effect of the GFD in patients with IBS is an unspecific consequence of reducing FODMAPs intake, given that wheat is one of the possible sources of FODMAPs.

\section{Clinical Picture and Diagnosis}

So far, NCGS has been described only in adults, and no data are available for the pediatric population. We have recently described a series of 15 children (M: 10; median age: 10, 3 years, range 1, 6-15 years) diagnosed as having NCGS in tertiary referral centers for CD. All children were referred for a clear association of wheat ingestion and symptoms to confirm/exclude an adverse food reaction [12]. For comparison, 15 children with active CD diagnosed according to the European Society of Pediatric Gastroenterology, Hepatology, and Nutrition criteria [23], and 15 control children enrolled from the children followed in our outpatient clinic for functional disorders [24] without $\mathrm{CD}$ and with no association between symptoms and any particular food were recruited.

After exclusion of CD and WA to confirm the diagnosis of NCGS, a "single blind parent-aware" gluten challenge was performed. Before the challenge, gluten had been withdrawn from the patient's diet for at least 8 weeks, and the challenge was performed in a hospital setting by administering gluten-containing food in increasing doses until a cumulative dose of at least $5 \mathrm{~g}$ was reached after $24 \mathrm{~h}$. The children were asked to record all symptoms in a diary provided, and children with a $30 \%$ increase in scores were considered to have a positive challenge.

In our series, the clinical presentation was characterized by typical gastrointestinal symptoms, such as abdominal pain and chronic diarrhea (Table 1). In this respect, this condition is similar to $\mathrm{CD}$ or functional disorders and therefore not suggestive specifically of NCGS. Interestingly, as compared to adults, the extra-intestinal manifestations were less frequent, the most common extraintestinal symptom being tiredness; none complained of behavioral changes, "foggy mind," eczema and/or rash, muscle cramps, leg numbness, and weight loss as reported in adults. Unlike adult patients, NCGS in children was more common in males.

As well as adults, children lack a biochemical/serological/genetic marker of this condition. Native AntiGliadin Antibody (AGA) IgG was the most frequently detected antibody, found in $66 \%$ of children with NCGS although with titers significantly lower in children with GS compared with those with CD. HLA typing showed the presence of DQ2 in 7 of 15 of children with NCGS (46\%). No difference was found in the determination of nutritional, biochemical, and inflammatory markers between 
Table 1 Demographic, clinical, serological, genetic, and histological data of the 15 GS children

\begin{tabular}{|c|c|c|c|c|c|c|}
\hline $\mathrm{P}$ & Sex & Symptoms & $\begin{array}{l}\text { Time to } \\
\text { relapse }(\mathrm{h})\end{array}$ & CD serology & $\begin{array}{l}\text { HLA- } \\
\text { DQ2/8 }\end{array}$ & Histology \\
\hline Case 1 & M & Chronic diarrhea & 40 & All negative & Present & $\begin{array}{l}\text { Not } \\
\text { available }\end{array}$ \\
\hline Case 2 & M & Chronic diarrhea, failure to thrive & 38 & AGA IgG positive & Absent & Marsh 0 \\
\hline Case 3 & M & Chronic diarrhea & 80 & AGA-IgG positive & Present & Marsh 0 \\
\hline Case 4 & M & Abdominal pain, chronic diarrhea & 38 & AGA-IgG/IgA positive & Absent & Marsh 0 \\
\hline Case 5 & $\mathrm{~F}$ & Abdominal pain, diarrhea/constipation, limb pain & 45 & All negative & Absent & Not available \\
\hline Case 6 & M & Abdominal pain, chronic diarrhea, vomiting, headache & 44 & All negative & Present & Marsh 1 \\
\hline Case 7 & M & Abdominal pain, chronic diarrhea, & 39 & All negative & Absent & Marsh 0 \\
\hline Case 8 & M & Abdominal pain, tiredness, vomiting, headache, limb pain & 40 & AGA-IgG positive & Absent & Not available \\
\hline Case 9 & $\mathrm{~F}$ & Abdominal pain & 44 & AGA-IgG positive & Present & Marsh 0 \\
\hline Case 10 & $\mathrm{~F}$ & $\begin{array}{l}\text { Abdominal pain, failure to thrive, tiredness, bloating } \\
\text { constipation, vomiting }\end{array}$ & 40 & AGA-IgG positive & Present & Marsh 0 \\
\hline Case 11 & M & Abdominal pain, bloating, chronic diarrhea, tiredness & 46 & AGA-IgG positive & Present & Marsh 0 \\
\hline Case 12 & $\mathrm{~F}$ & Abdominal pain, chronic diarrhea, bloating, tiredness & 47 & AGA-IgG positive & Absent & Marsh 1 \\
\hline Case 13 & M & Abdominal pain, constipation & 43 & All negative & Present & Marsh 0 \\
\hline Case 14 & M & Abdominal pain, bloating, chronic diarrhea, headache & 49 & AGA-IgG positive & Absent & Not available \\
\hline Case 15 & $\mathrm{~F}$ & Abdominal pain, chronic diarrhea, tiredness, limb pain & 55 & AGA-IgG positive & Absent & Marsh 0 \\
\hline
\end{tabular}

NCGS and controls. Similarly to adults [25], the positivity of native AGA-IgG and the presence of DQ2 were more common (about half of the cases) in NCGS than control children (Table 2). Of note, AGA IgG disappears in adult NCGS patients after six moths of GFD [26], while we do not have data on our pediatric population.

Histologic analysis revealed a normal to mildly inflamed mucosa in children with NCGS: $82 \%$ were classified as Marsh 0 and $18 \%$ were classified as Marsh 1. Intraepithelial Lymphocytes (IEL) counts were significantly lower in the children with NCGS compared with those with CD. Villanacci et al. [27] observed in adults a peculiar distribution of IEL with two morphological aspects: (a) cluster of $\mathrm{T}$ lymphocytes in the superficial epithelium and (b) linear disposition of $\mathrm{T}$ lymphocytes in the deeper part of the mucosa above the muscularis mucosae.

As mentioned, the lack of serological or histological markers makes the diagnosis of NCGS a clinical-only one, based on the exclusion of CD and WA and on gluten challenge.

However, open-food challenges are obviously open to the possible confounding effect of placebo/nocebo caused by gluten exclusion/consumption: thus, DBPCFC gluten challenge should be considered the best option to confirm NCGS diagnosis. In spite of this, current guidelines accept an open-food challenge [28].

As it is unknown, if NGCS is a permanent or transitory condition, periodical reassessments (e.g., every 6-12 months) are strongly recommended [2].

\section{Is it Really a New Entity?}

As previously mentioned, NCGS was originally described in the 1980s [3] and many authors observed children and adult patients with chronic diarrhea, bloating, abdominal pain, nausea, headaches, with normal small intestinal biopsy specimens, and the absence of CD antibodies who responded dramatically to a GFD [29].

In pediatrics, NCGS might have been diagnosed as a non-IgE-mediated adverse reaction to wheat. In fact, the possible overlapping between NCGS and delayed allergic reaction to wheat proteins is still an open question. NonIgE-mediated reactions tend to be delayed, with the onset of symptoms occurring from $1 \mathrm{~h}$ to several days after ingestion of food. A significant proportion of children with food allergy do not have circulating food proteinspecific IgE and show negative results to skin prick tests [30]. At present, there is no diagnostic test for non-IgEmediated adverse reactions to food. Atopy Patch Tests (APTs) are not standardized and are not recommended for routine use by current guidelines [31]; they might nevertheless be of help in excluding food allergy-related gastrointestinal symptoms, particularly for wheat allergy [32, 33].

Furthermore, previous studies on food allergy include cases with clinical characteristics resembling NCGS. In 2006, Venter et al. [34] established the prevalence of sensitization to food allergens using food challenges among all 6-year-old children living on the Isle of Wight. 
Table 2 Serological and hematological parameters of children with functional disorders, gluten sensitivity, and celiac disease

\begin{tabular}{lllc}
\hline & Controls & Gluten sensitivity & Celiac disease \\
\hline AGA-IgG $(<10 \mathrm{UA} / \mathrm{ml})$ & $4.5 \pm 4^{*}$ & $21.6 \pm 21.7^{*}$ & $87.4 \pm 81.3$ \\
AGA-IgG positivity $n(\%)$ & $2(13)^{\mathrm{a}}$ & $10(66)^{\mathrm{a}}$ & $13(86)$ \\
AGA-IgA $(<10 \mathrm{UA} / \mathrm{ml})$ & $4.1 \pm 2.5^{\mathrm{a}}$ & $2.6 \pm 2.7^{\mathrm{a}}$ & $46.5 \pm 58.8$ \\
AGA-IgA positivity $n(\%)$ & $0^{\mathrm{a}}$ & $1(6.7)^{\mathrm{a}}$ & $11(73)$ \\
TTG-IgG $(<10 \mathrm{UA} / \mathrm{ml})$ & $3.9 \pm 3.6^{\mathrm{a}}$ & $2.0 \pm 0.8^{\mathrm{a}}$ & $28.5 \pm 28$ \\
TTG-IgG positivity $n(\%)$ & $1(6.7)^{\mathrm{a}}$ & $0^{\mathrm{a}}$ & $12(80)$ \\
TTG-IgA $(<10 \mathrm{UA} / \mathrm{ml})$ & $0.6 \pm 0.6^{*}$ & $1.4 \pm 0.7^{*}$ & $137.6 \pm 78.6$ \\
Hemoglobin $(>12 \mathrm{~g} / \mathrm{dl})$ & $12.5 \pm 0.8^{\mathrm{a}}$ & $12.8 \pm 1^{\mathrm{a}}$ & $12 \pm 1$ \\
Iron $(>30 \mathrm{ng} / \mathrm{ml})$ & $92.2 \pm 36^{*}$ & $57 \pm 26.2^{*}$ & 0.001 \\
Ferritin $(>15 \mathrm{ng} / \mathrm{ml})$ & $25.8 \pm 6.8^{\mathrm{a}}$ & $31.0 \pm 14.8^{\mathrm{a}}$ & 0.001 \\
GOT $(<30 \mathrm{U} / \mathrm{ml})$ & $15.9 \pm 4^{\mathrm{a}}$ & $18.3 \pm 9^{\mathrm{a}}$ & $15.5 \pm 8.7$ \\
Erythrocyte sedimentation rate $(<20 \mathrm{~mm} / \mathrm{h})$ & $7.5 \pm 3.9^{\mathrm{a}}$ & $8.0 \pm 2.9^{\mathrm{a}}$ & $26.7 \pm 8.8$ \\
\hline
\end{tabular}

$* p<0.004$

${ }^{\mathrm{a}} p=\mathrm{NS}$

One of the children described by Venter presenting with diarrhea and abdominal pain showed negative skin prick tests and positive wheat food challenge [34]. In a Finnish study [35] published in 2010 on the prognosis of wheat hypersensitivity, the authors describe four children with gastrointestinal symptoms related to gluten and confirmed by an open wheat challenge who were diagnosed as allergic to wheat despite a negative SPT to gluten and gliadin [35]. Finally, in a retrospective study [36] on 437 children with food protein-induced gastrointestinal symptoms, $41.7 \%$ were managed successfully with a multiple elimination diet including wheat. This study, however, in addition to children with non-IgE-mediated gastrointestinal food allergies, also included children with allergic enteropathy and enterocolitis [36].

In children, the differential diagnosis between NCGS and non-IgE-mediated adverse reactions to wheat resides mainly on clinical observations: although more studies are needed, some considerations argue in favor of NCGS as a distinct clinical entity even in children.

Age appears to be a main criterion. In fact, nearly all children with wheat hypersensitivity become tolerant by adolescence: wheat tolerance is acquired by $59,69,84$, and $96 \%$, by age $4,6,10$, and 16 , respectively [35]. Thus, the older the child, the less the chances of a non-IgE-mediated adverse reactions to wheat. Children in our series were older than the typical wheat hypersensitive child (66\% older than 10 years).

Furthermore, a majority of wheat allergic children suffer from other allergic stigmata such as positive family history for atopy, atopic dermatitis, or other food allergies [37]. As a consequence, the absence of these other factors would support NCGS: in our study, none of the patients with
NCGS had atopic dermatitis, personal or family history of atopy, or wheat-dependent exercise-induced anaphylaxis.

\section{What Next?}

Currently, NCGS is surrounded by skepticism and surely there is more popular talking rather than scientific data, if we compare the citations in a web browser as opposed to Pubmed [38]. However, it is indeed the limited scientific literature on the topic that generates all the doubts. In the era of evidence-based medicine, there is a strong need for validated biomarkers to support a diagnosis not based on clinical subjective criteria. In their absence, several problems need to be addressed, among them first and foremost what type of rigorous diagnostic double-blind placebocontrolled process must be followed, in the absence of a valid biomarker: the type of antigen used, the dose, and the time of its administration, the way symptoms are reported and scored.

High priorities in the research agenda of NCGS are

- Reach an unequivocal definition for this syndrome.

- Define if indeed is gluten (or gluten alone) the real trigger for NCGS.

- Characterize the role of FODMAP: sometimes or always responsible?

- Understand how rigorous the exclusion of gluten has to be for NCGS patients.

- Are all types of wheat involved in NCGS? And what about rye and barley?

- Should gluten-free diet be continued for life? 
- Should we include NCGS in the classification of adverse food reaction in pediatrics? [39].

\section{Conclusions}

NCGS, mainly in pediatrics, is an entity awaiting validation, diagnostic, and clinical definition and possibly pathogenic mechanism clarification.

There is a need of "separating the wheat from the chaff" [40] and this can be achieved only with proper scientific information, and prospective, multi-center studies addressing the many unsolved issues on NCGS.

Until a specific biomarker of this condition is available, NCGS should be suspected only in selected children after a proper exclusion of CD and WA. Unmotivated gluten-free diet could be dangerous not only because of its cost but also for the risk to classify incorrectly patients.

Acknowledgments I would like to express my very great appreciation to Lucia Verzillo for his precious work with patients reported in this review and a special thanks is extended to Dr. Maria Stella staff for the great help for the nutritional advice.

Disclosure Fernanda Cristofori, Francesca Arezzo, Antonia Gentile, and Ruggiero Francavilla declare that they have no conflict of interest.

Human and Animal Rights and Informed Consent This article does not contain any studies with human or animal subjects performed by any of the authors.

\section{References}

Papers of particular interest, published recently, have been highlighted as:

- Of importance

-• Of major importance

1. Sapone A, Bai JC, Ciacci C, et al. Spectrum of gluten-related disorders: consensus on new nomenclature and classification. BMC Med. 2012;10:13.

2. - Catassi C, Bai JC, Bonaz B et al. Non-celiac gluten sensitivity: the new frontier of gluten related disorders. Nutrients 2013; 5(10):3839-53. Report of Munich consensus conference with review of the recent lecterature on NCGS.

3. Cooper BT, Holmes GK, Ferguson R, et al. Gluten-sensitive diarrhea without evidence of celiac disease. Gastroenterology. 1980;79:801-6.

4. Ellis A, Linaker BD. Non celiac gluten sensitivity? Lancet. 1978;1(8078):1358-9.

5. Simpson S, Lebwohl B, Di Giacomo D, et al. Awareness of celiac disease and gluten sensitivity in US. Gastroenterology. 2011;140:S443.

6. Painter K. Gluten-free diets gaining in popularity. USA Today, August 2008.
7. Tanpowpong P, Ingham TR, Lampshire PK, et al. Celiac disease and gluten avoidance in New Zealand children. Arch Dis Child. 2012;97:12-6.

8. Sapone A, Lammers KM, Mazzarella G, et al. Differential mucosal IL-17 expression in two gliadin-induced disorders: gluten sensitivity and the autoimmune enteropathy celiac disease. Int Arch Allergy Immunol. 2010;152(1):75-80.

9. - Biesiekierski JR, Newnham ED, Irving PM, et al. Gluten causes gastrointestinal symptoms in subjects without celiac disease: a double-blind randomized placebo-controlled trial. Am J Gastroenterol. 2011;106:508-14. First demonstration of the existence of NCGS with DBPCFC.

10. - Carroccio A, Mansueto P, Iacono G. Non-celiac wheat sensitivity diagnosed by double-blind placebo-controlled challenge: exploring a new clinical entity. Am J Gastroenterol. 2012; 107(12):1898-906. This large retrospective study show that 30\% of IBS patient present a positive wheat challenge.

11. Vasquez Roque MI, Camilleri M, Smyrk T, et al. A controlled trial of gluten free diet in patients with irritable bowel syndromediarrhoea: effects on bowel frequency and intestinal function. Gastroenterology. 2013;144(5):903-11.

12. •• Francavilla R, Cristofori F, Castellaneta S. Clinical, serologic, and histologic features of gluten sensitivity in children. J Pediatr. 2014;164(3):463-67. This is the first study describing a series of pediatric cases of GS and supporting the existence of this condition in children across all ages.

13. Carroccio A, Rini G, Mansueto P. Non-celiac wheat sensitivity is a more appropriate label than non-celiac gluten sensitivity. Gastroenterology. 2014;146(1):320-1.

14. Tanpowpong P, Broder-Fingert S, Katz AJ, et al. Predictors of gluten avoidance and implementation of a gluten-free diet in children and adolescents without confirmed celiac disease. J Pediatr. 2012;161(3):471-5.

15. Digiacomo DV, Tennyson CA, Green PH, et al. Prevalence of gluten-free diet adherence among individuals without celiac disease in the USA: results from the Continuous National Health and Nutrition Examination Survey 2009-2010. Scand J Gastroenterol. 2013;48(8):921-5.

16. Aziz I, Lewis NR, Hadjivassiliou M, et al. A UK study assessing the population prevalence of self-reported gluten sensitivity and referral characteristics to secondary care. Eur J Gastroenterol Hepatol. 2014;26(1):33-9.

17. Sapone A, Lammers KM, Casolaro V, et al. Divergence of gut permeability and mucosal immune gene expression in two glutenassociated conditions: celiac disease and gluten sensitivity. BMC Med. 2011;9:23.

18. Brottveit M, Beitnes AC, Tollefsen S, et al. Mucosal cytokine response after short-term gluten challenge in celiac disease and nonceliac gluten sensitivity. Am J Gastroenterol. 2013;108(5):842-50.

19. Verdu EF, Huang X. Natividad J Gliadin-dependent neuromuscular and epithelial secretory responses in gluten-sensitive HLADQ8 transgenic mice. Am J Physiol Gastrointest Liver Physiol. 2008;294:G217-25.

20. Bucci C, Zingone F, Russo I, et al. Gliadin does not induce mucosal inflammation or basophil activation in patients with nonceliac gluten sensitivity. Clin Gastroenterol Hepatol. 2013; 11(10):1294-9.

21. Junker $\mathrm{Y}$, Zeissig S, Kim SJ, et al. Wheat amylase trypsin inhibitors drive intestinal inflammation via activation of toll like receptor 4. J Exp Med. 2013;209:2395-408.

22. Biesiekirski JR, Peters SL, Newnham ED, et al. No effects of gluten in patients with self-reported non-celiac gluten sensitivity following dietary reduction of low-fermentable, poorly absorbed, short-chain carbohydrates. Gastroenterology. 2013;145:320-32.

23. Husby S, Koletzko S. Korponay-Szabó IREuropean Society for Pediatric Gastroenterology, Hepatology, and Nutrition guidelines 
for the diagnosis of celiac disease. J Pediatr Gastroenterol Nutr. 2012;54(1):136-60.

24. Rasquin A, Di Lorenzo C, Forbes D, et al. Childhood functional gastrointestinal disorders: child/adolescent. Gastroenterology. 2006;130:1527-37.

25. Volta U, Tovoli F, Cicola R, et al. Serological tests in gluten sensitivity (nonceliac gluten intolerance). J Clin Gastroenterol. 2012;46(8):680-5.

26. Caio G, Volta U, Tovoli F, et al. Effect of gluten free diet on immune response to gliadin in patients with non-celiac gluten sensitivity. BMC Gastroenterol. 2014;14(1):26.

27. Villanacci V, Lanzini A, Lanzarotto F, et al. Observations on the paper of Carroccio et. al. Non-celiac wheat sensitivity diagnosed by double-blind placebo-controlled challenge: exploring a new clinical entity. Am J Gastroenterol. 2013;108(4):619-20.

28. Boyce JA, Assa'ad A, Burks AW, et al. Guidelines for the diagnosis and management of food allergy in the United States: report of the NIAID-sponsored expert panel. J Allergy Clin Immunol. 2010;126(6):1105-18.

29. Rúa EC, Drut R, Peña AS. Non-celiac wheat sensitivity is not a new entity. Am J Gastroenterol. 2013;108(11):1805.

30. Sicherer SH, Sampson HA. Food allergy: epidemiology, pathogenesis, diagnosis, and treatment. J Allergy Clin Immunol. 2014;133(2):291-307.

31. Sicherer SH, Sampson HA. Food allergy. J Allergy Clin Immunol. 2010;125(Suppl 2):S116-25.
32. Majama H, Moiso P, Holm K, et al. Wheat allergy: diagnostic accuracy of Skin Prick test and Patch test and specific IgE. Allergy. 1999;54(8):851-6.

33. Canani RB, Ruotolo S, Auricchio L, et al. Diagnostic accuracy of the atopy patch test in children with food allergy-related gastrointestinal symptoms. Allergy. 2007;62(7):738-43.

34. Venter C, Pereira B, Grundy J, et al. Prevalence of sensitization reported and objectively assessed food hypersensitivity amongst six-year-old children: a population-based study. Pediatr Allergy Immunol. 2006;17(5):356-63.

35. Kotaniemi-Syrjanen A, Palosuo K, Jartti T, et al. The prognosis of wheat hypersensitivity in children. Pediatr Allergy Immunol. 2010;21:e421-8.

36. Meyer R, Fleming C, Dominguez-Ortega G, et al. Manifestations of food protein induced gastrointestinal allergies presenting to a single tertiary paediatric gastroenterology unit. World Allergy Organ J. 2013;6(1):13.

37. Inomata N. Wheat allergy. Curr Opin Allergy Clin Immunol. 2009;9(3):238-43.

38. Di Sabatino A, Corazza GR. Nonceliac gluten sensitivity: sense or sensibility? Ann Intern Med. 2012;156(4):309-11.

39. Guandalini S, Newland C. Differentiating food allergies from food intolerances. Curr Gastroenterol Rep. 2011;13(5):426-34.

40. Sanders DS, Aziz I. Non celiac wheat sensitivity: separating the wheat from the chat! Am J Gastroenterol. 2012;107(12):1908-12. 